\title{
DOPPLER IMAGES OF HR 1099 FROM 1981-1993
}

\author{
S. S. VOGT \\ UCO/Lick Observatory, \\ Univ. of California, \\ Santa Cruz, U.S.A. \\ AND \\ A. P. HATZES \\ McDonald Observatory, \\ Univ. of Texas, \\ Austin, U.S.A.
}

\begin{abstract}
We present 23 Doppler images of the spotted RS CVn star HR 1099 (V711 Tau) from 1981 to 1993. All of the images show a large variable-shape cool spot straddling the pole. Many of the images also show a number of isolated low-latitude spots. This image set has been used to track the emergence and evolution of spots on this star over the 12-year interval. The Doppler images are compared to previous 'few-circular-spot' photometric model solutions when available. These photometric solutions do not well-represent the spot distribution. We discuss, among other things: the location on the stellar surface where spots first appear, their subsequent movement with time, differential rotation from spot motions, and spot cycles and dynamos within HR 1099.
\end{abstract}

\section{Introduction}

We have been monitoring HR 1099 using Doppler imaging for the past 15 years in an effort to better understand the nature of the large dark spots on RS CVn stars. We present here only a very brief summary of an extensive paper now in preparation concerning Doppler imaging of HR 1099 from 1981 - 1993. The length limitation of this symposium paper allows only a few highlights to be summarized here. For further details, the reader is referred to our forthcoming paper (Vogt, Hatzes, Misch, and Kürster 1996). 

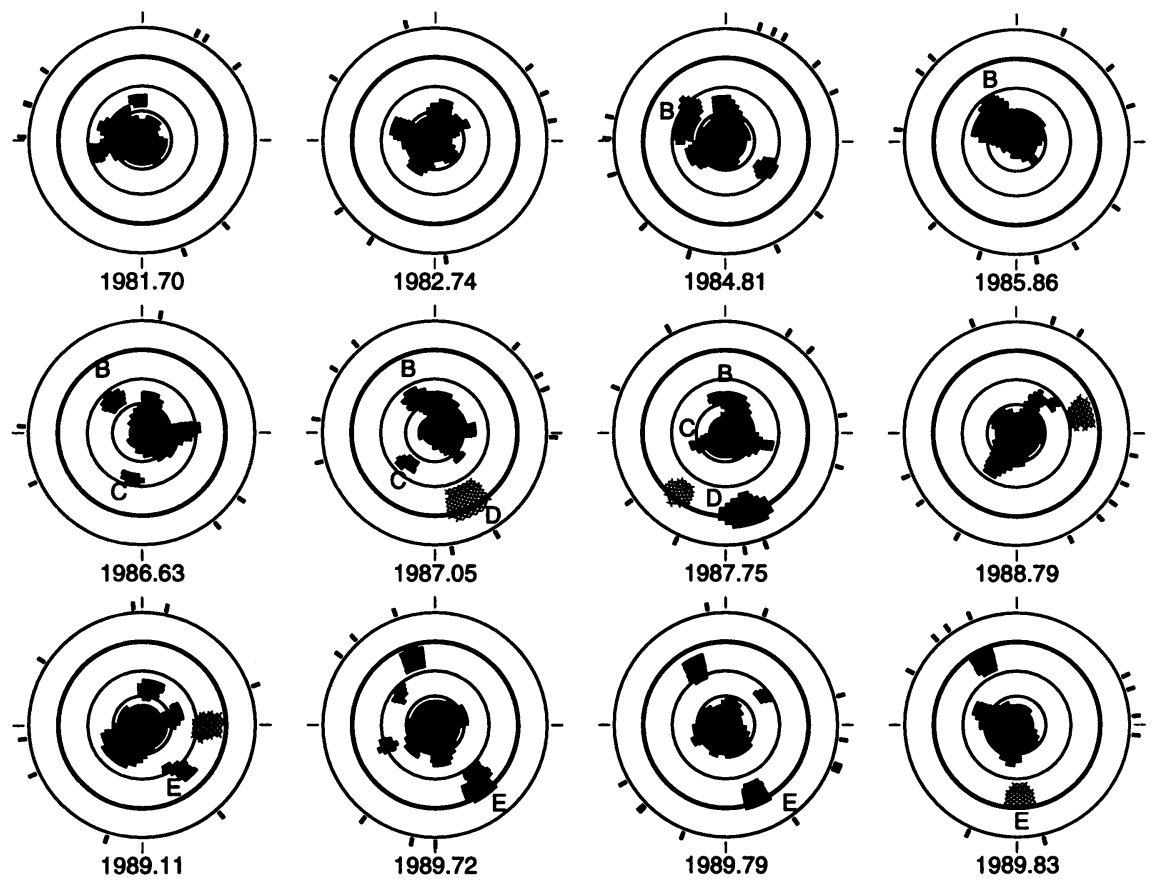

Figure 1. Doppler images of HR 1099 from 1981 through 1989. Below each image is the mid-epoch of the image. The heavy solid line represents the stellar equator and the short radial tick marks indicate the phases of the observations. Cross-hatched regions indicate spot features (hot, cool, or rapidly-moving spots) not found in the Doppler images but suggested by contemporaneous light curves. The hatched spot in the 1987.75 image represents the location of spot D in 1988.07

\section{The Doppler images}

Doppler images for HR 1099 spanning the interval 1981.70 to 1993.15 are shown in Figures 1 and 2. The images were derived using the maximum entropy method (Vogt, Penrod, and Hatzes 1987) and are shown in flattened polar projection down to a latitude of $-30^{\circ}$. For clarity, each image is shown as a thresholded 2-temperature distribution wherein pixels below the threshold value (about $500 \mathrm{~K}$ cooler than the photosphere) are shown as spot (black). The predicted spectral line flux profiles from these thresholded images are consistent with all the observed profiles. For the most part, these 2-temperature versions of the Doppler images also provided an adequate fit to contemporaneous light curves. 


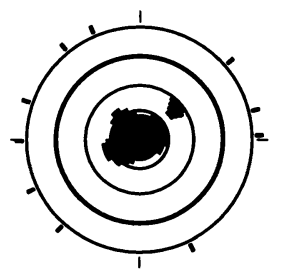

1990.69
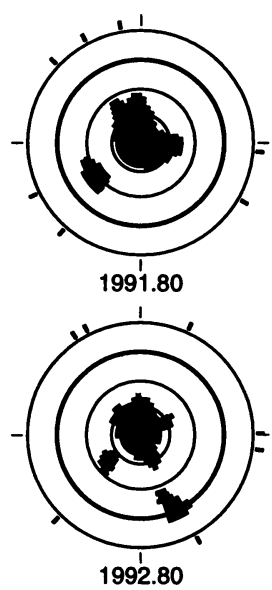
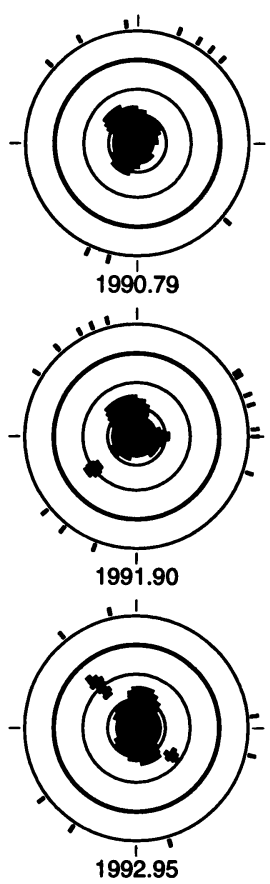
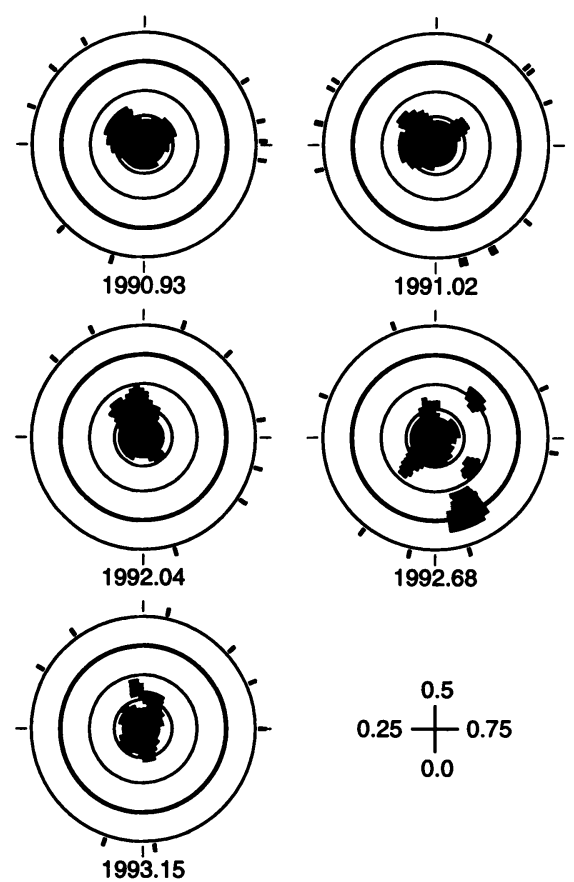

Figure 2. Doppler images for HR 1099 from 1990 to 1993. The phase convention is shown in the lower right corner.

\section{Comparison of the images with 'few-circular-spot' solutions}

Most spotted stars rotate too slowly to be amenable to Doppler imaging. In these cases, one must resort to fitting light curves with simple models involving 2 or 3 circular spots. However, these simple spot models are known to be quite non-unique since a light curve alone does not offer much constraint for the large set of possible image solutions. It was therefore of particular interest to compare the results of such 'few-circular-spot' models against the more detailed Doppler imagery of HR 1099. This comparison would reveal how well these simple models actually did, and thereby help us to better assess their effectiveness for the slowly-rotating spotted stars.

In the literature we were able to find contemporaneous "few-circularspot" solutions (from light curve fitting) only for our $1981.70,1982.74$, and 1989.88 images. These solutions are shown in Figure 3 as circles overlaid on gray-scale versions of our Doppler images. Images (3a) and (3b) show the solutions for 1981.70 of Rodono et al. (1986) (photometry taken at epoch 1981.7) and Kang and Wilson (1989) (photometry taken at epoch 1981.9), respectively. Image (3c) shows the solution for 1982.74 of Andrews et al. (1988) (photometry taken at epoch 1983.0), and image (3d) the solution 

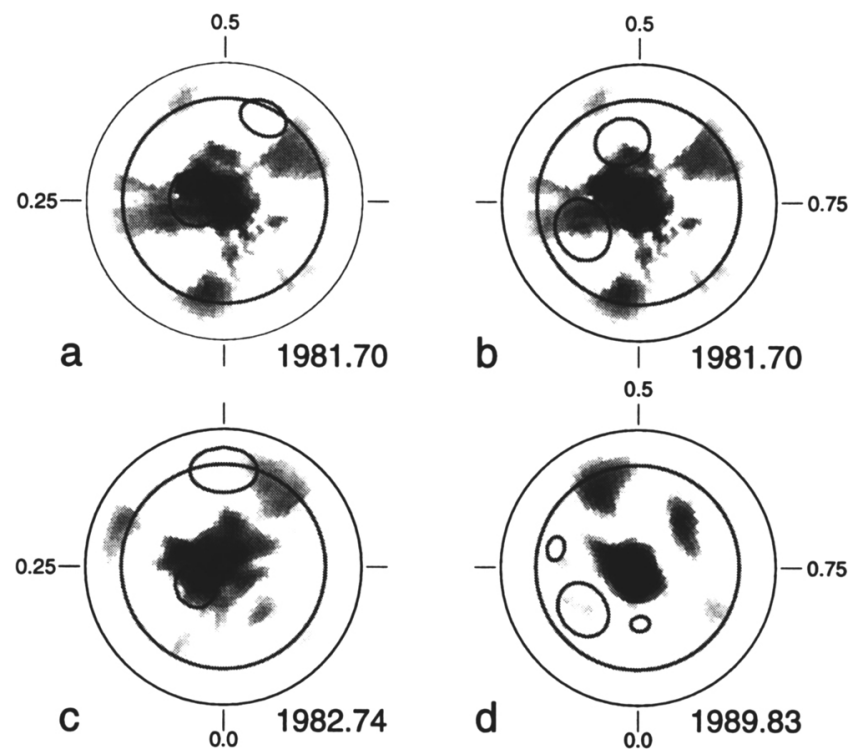

Figure 3. 'Circular-spot' photometric solutions (circles) derived from contemporaneous light curves and superimposed on the Doppler images. The circle nearest phase 0.0 in image (d) represents a hot spot.

for 1989.88 of Zhai et al. (1994) (photometry taken at epoch 1989.96). The spot near phase 0.0 for this last photometric solution represents a hot spot about $750 \mathrm{~K}$ hotter than the photosphere.

The Doppler images and 'few-circular-spot'solutions both adequately fit the broadband light curves, with the main difference being that the Doppler images also fit a set of line flux profiles and are thus much more highly constrained. Unfortunately, the agreement between these 'few-circular-spot' solutions and the Doppler images is not very encouraging, nor is the agreement between the circular-spot solutions of different researchers for the same epoch. Clearly, the light curves alone just do not contain enough information to adequately constrain the spot solutions.

Some instances of correspondence of features do exist. For instance, the phase 0.25 spot of the 1981.70 solution of Rodono et al. (1986) (Figure 3a) does coincide well with the major protuberance on the polar spot at that epoch. And one of the spots in the 1982.74 solution (Andrews et al. 1988) does coincide with a major polar spot protuberance at phase 0.15 . Also, in the 1989.83 image the hot spot found near phase 0.0 (Zhai et al. 1994) does appear to coincide well with a spot we show as Feature $\mathrm{E}$ at that epoch in Figure 1. This spot did not appear in our raw Doppler image (Fig. 3), as we are imaging only dark features, but did appear (Fig. 1) after we added 
the light curve constraints to the image.

On the other hand, many of the spots predicted by these models do not seem to coincide with any feature of the image. Also, as expected, none of the spot models detect the presence of the polar spot. So, while some instances of correspondence can be found, these circular-spot solutions do not seem to yield much useful information on the spot distribution. In fact, for all the cases presented in Figure 3, these solutions are quite misleading.

Conversely, agreement of Doppler images of HR 1099 among different researchers is generally quite excellent. Good examples are comparisons of our 1988.79 and 1990.93 images with the Doppler images of Donati et al. (1992). Here, most of the subtle details of the shape of the polar spot are faithfully reproduced between the two research groups, and these images were derived from completely independent data sets, with different imaging software. Feature E at about phase 0.9 in our 1989.72 and 1989.79 images also coincides closely with the large monopolar $985 \mathrm{G}$ region detected at phase 0.855 in Zeeman Doppler imaging by Donati et al. (1990) and may indicate that at least some starspots are associated with large unipolar regions.

\section{Tracking the emergence and migration of spots}

One fundamental result revealed by the image set is that major spots frequently emerge (first appear) at low latitudes. Features D and E are two good examples of this; both first appear essentially at the equator. Other equatorial and low-latitude spot emergences are also seen. This result contradicts the theoretical explanation of polar spots put forth by Schüssler and Solanki (1992). In their model, coriolis forces cause buoyant magnetic flux generated deep within the star to rise along paths parallel to the star's rotation axis rather than along radial paths. Their model then predicts that flux should erupt only at high latitudes, thereby perhaps explaining the ubiquitous polar spots on these stars. However, the common-place emergence of low-latitude spots on the very rapid rotator HR 1099 is in direct contradiction to this prediction, and may be a problem for the Schüssler and Solanki model.

It has also often been assumed that the 'migrating photometric waves' in the light curves of RS CVn stars are attributable to longitudinal migration of spots on a differentially-rotating star. The phase drift of spotted star light curves is an easily and accurately measurable quantity, and attempts have been made to derive the differential rotation of spotted stars from such light curve phase drifts. In the presence of latitudinal shear, spots at different latitudes would have different rotation periods, migrate in longitude with respect to the orbit, and thus cause the light curve to migrate in phase 


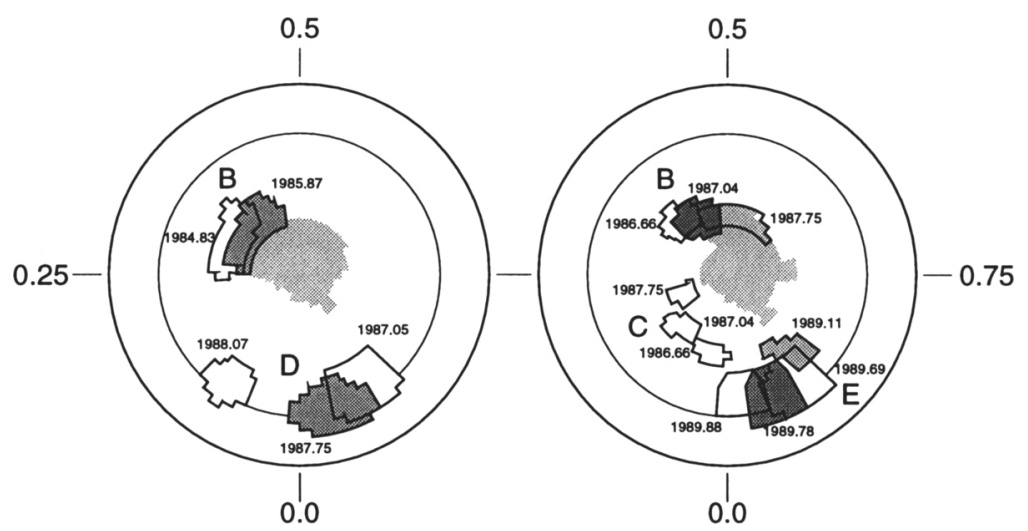

Figure 4. The migration tracks for spot features B, C, D, and E.

accordingly. In this simple picture, spots appearing above or below some intermediate co-rotation latitude (i.e. like the solar butterfly diagram for sunspots) could then cause the photometric light curve minimum to migrate in either direction.

Our imagery reveals that, at least for HR 1099, the situation is much more complex. The phase of the photometric minimum is affected strongly by the appearance and disappearance of a number of relatively-fixed large spots, as well as by movements of some of these spots, and also by changes in the shapes and areas of these spots. It is clearly not valid to invert these phase drifts through as simple a parameterization as longitude migration of one or two spots; the situation is much more complex. The phase of the migrating wave by itself reveals essentially nothing about the details of the differential rotation pattern on HR 1099.

Most spots come and go too quickly to track reliably with on-average one image per year. However, there were a number of isolated long-lived spots which we were able to track from year-to-year. Figure 4 shows the tracks of Features B, C, D, and E in the frame of reference of the orbit. Thus all migrations are occuring with respect to the orbital frame, with the secondary (hotter) star fixed at phase 0.5 . The position of spot $\mathrm{D}$ in 1988.07 was inferred from the photometry. A spot was required at that position for the predicted photometry from our 1987.75 Doppler image to fit the 1988.07-epoch photometry (Mohin and Raveendran 1993).

Feature B, at high latitude, lasted for almost 3 years and was seen migrating clockwise and northward very slowly with respect to the orbital frame. It apparently merged with the polar spot by 1987.75 . Feature C, at intermediate latitude, also migrated clockwise (angular velocity slower than the orbit) but faster relative to the orbit than Feature B. It also followed a northward-drifting clockwise spiral path finally merging with the polar 
spot. Features D and E also drifted clockwise, but yet even faster relative to the orbit than B or C.

The tracks of both Features B and C suggest that some spots which emerge at low or intermediate latitude do make their way up to the pole, and then apparently merge with the polar spot. Since the dark spots presumably trace magnetic flux, this must mean that some of the magnetic flux which emerges at lower latitudes then spirals pole-ward on HR 1099 in a clockwise manner (slower than the orbit) and merges eventually with the polar spot. It is not yet clear whether this flux is of the same or opposite polarity to the polar spot, and thus whether these northward-migrating low-latitude spots reinforce or cancel the polar spot field.

Feature B also appeared to get somewhat stretched in longitude as it approached the polar spot, and its overall track is quite reminiscent of the annulus of toroidal field found (Donati et al. 1992) encircling the polar spot of HR 1099 in 1990.9. Perhaps spots such as Feature B become sheared in longitude as they approach the pole, and thereby contribute to an annular ring of strong field around the polar spot. Feature B may thus be showing us directly how field lines get wrapped around the pole into the toroidal structure seen by Donati et al. 1992). Perhaps then, the 'polar spot' is simply that region where the field lines get wrapped sufficiently tightly around the pole, like spaghetti around a fork, to create fields strong enough to suppress convection in the photosphere, and thus create the cool polar spots.

\section{The differential rotation of HR $\mathbf{1 0 9 9}$}

The migration rates of these long-lived features were then used to derive the 'differential rotation' or rotation period of the star as a function of latitude. Figure 5 shows the measured period vs. two simple parameterizations of latitude $(\ell)$. On the Sun, rotation period depends nearly linearly on $\sin ^{2} \ell$, and we have thus used that as the latitude parameter in the upper panel of Fig. 5. We find a marginally better correlation with $\sin \ell$ though the small number of data points and scatter in the data precludes deriving a more precise functional form at this point. Interestingly, the rotation period also correlates well (bottom panel of Fig. 5) with $\left(1+3 \sin ^{2} \ell\right)^{1 / 2}$, an abscissa proportional to the surface strength of a centered axisymmetric magnetic dipole field. Given also that an extrapolation of the observed rotation periods points to synchronous rotation at the pole, these observations may well be indicating that spot migration motions are dominated by a large aligned magnetic dipole within HR 1099 which is co-rotating with the orbital frame. The relatively high surface field strengths near the pole would then enforce near-corotation of high-latitude spot features, while the 
decreasing field strength with decreasing latitude would allow progressively faster 'slip' or clockwise longitude migration of spots, just as observed.

Whatever the precise form of latitudinal dependence of period, the basic picture which emerges is that spots at high latitudes on HR 1099 are rotating in synchronism with the orbit, and rotation period increases with decreasing latitude. The equator is thus rotating more slowly than the poles, opposite to the solar case. And while the Sun shows a $20 \%$ difference between polar and equatorial angular velocity (in the photosphere from sunspots), HR 1099 shows only about a $0.4 \%$ difference with its starspots. This is a factor of about 50 less than from photospheric sunspot motions, but similar to the small differential rotation values observed in solar coronal holes. Given that starspots also bear a striking morphological resemblance to solar coronal holes, this is perhaps not altogether surprising. It is consistent with the basic picture that starspots are essentially just coronal hole-like structures (footprints of the unipolar magnetic regions of a current-free global coronal magnetic field), but where the global field is multi-kgauss-strength rather than the few-gauss-strength global fields of the Sun.

Our result of very small differential rotation for HR 1099 is in excellent accord with the results of Hall (1991) who found that differential rotation signatures (from light curve stability) of a large sample of spotted latetype stars decrease rapidly as angular velocity increases. Rapidly-rotating spotted stars such as HR 1099 thus appear to approach solid-body rotation (as far as spot movements are concerned). If, as we believe, there is a strong (multi-kgauss) axisymmetric magnetic dipole within the star (which is causing the large cool polar spots), it may well be expected to globally dominate the movements of these starspots, just as the current-free coronal field of the Sun does in the case of coronal hole structures (Wang and Sheeley 1993). A 5-10 kG dipole field strength at the pole would still be 2.5-5 kG strength at the equator, and probably strong enough to compete seriously with shearing forces of photospheric differential rotation. Newly emerged magnetic flux would be obliged to immediately connect with this powerful external global dipole field, and any tendency for shearing due to differential rotation would be strongly resisted by the current-free potential field. This picture might simply explain the observed trend toward solid-body rotation of starspots with rotation velocity. More rapid rotation gives rise to a stronger current-free global dipole field which, in turn, enforces increasingly solid-body rotation of the spots (the unipolar magnetic footprints of this field) as the rotational angular velocity increases. 

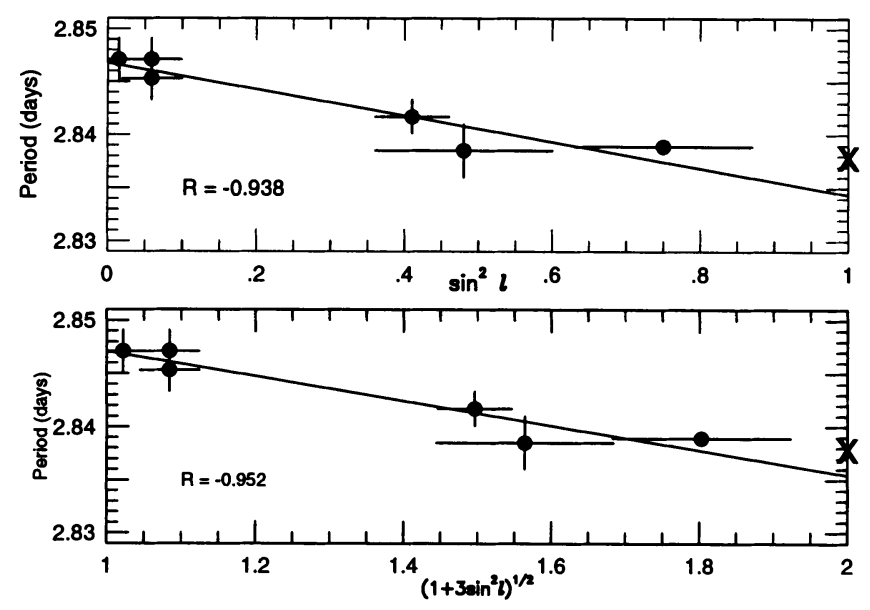

Figure 5. The rotation period of individual spot features as a function of $\sin ^{2} \ell$ [top] and $\left(1+3 \sin ^{2} \ell\right)^{1 / 2}$ [bottom]. The location of a synchronously-rotating pole is shown by the cross at the right edge of each plot.

\section{Spot/Activity cycles}

On the Sun, the area of the polar coronal hole varies dramatically and periodically, in anti-phase with the sunspot cycle. If starspots are stellar analogs of solar coronal hole structures, the best hope of detecting a dynamo cycle is probably then to look for periodicity in the area of the polar spot. The top panel of Figure 6 shows polar and low-latitude spot areas (percent of visible surface) as a function of time. There is an overall decrease in the polar spot area of about 11\% from 1982-1993 (the solid line represents a linear regression). The lower panel shows the mean brightness level for HR 1099 (Mohin and Raveendran 1993). The line represents a linear regression using the full span (1975-1991) of the mean light data and this yields an overall brightness increase of 0.045 mag over the 12-year span of the Doppler images. This observed increase agrees well with a predicted increase of 0.042 magnitudes (calculated from the Doppler images) and would seem to indicate that the mean light level here is, to first order, a good proxy of (polar) spot area.

A period analysis of the de-trended polar spot area shows maximum power at a period of $3.0 \pm 0.2 \mathrm{yr}$. The Scargle-type periodogram for the polar spot area is shown in the upper right of Figure 7. The false alarm probability (FAP) of the maximum peak is 0.08 . The top left panel of Figure 7 shows the percent changes in the polar spot area phased to this 3-year period.

A period analysis was also performed on the low latitude spot areas (bottom right panel of Fig. 7) and this yielded a period of $2.6 \pm 0.2 \mathrm{yr}$ with 


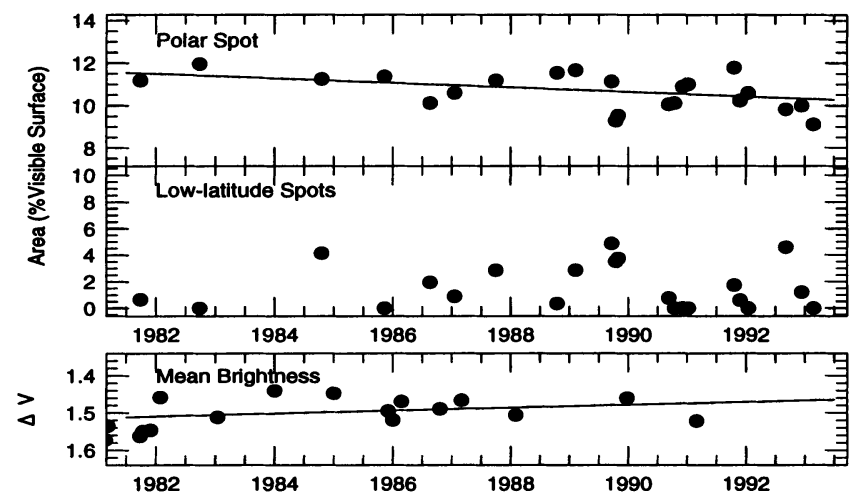

Figure 6. (Top) The polar spot area (percent of visible surface) as a function of year. The solid line represents a linear regression. (Middle) The yearly variation in the low-latitude spot area. (Bottom) The mean brightness level. The solid line represents a least squares fit to the trend.
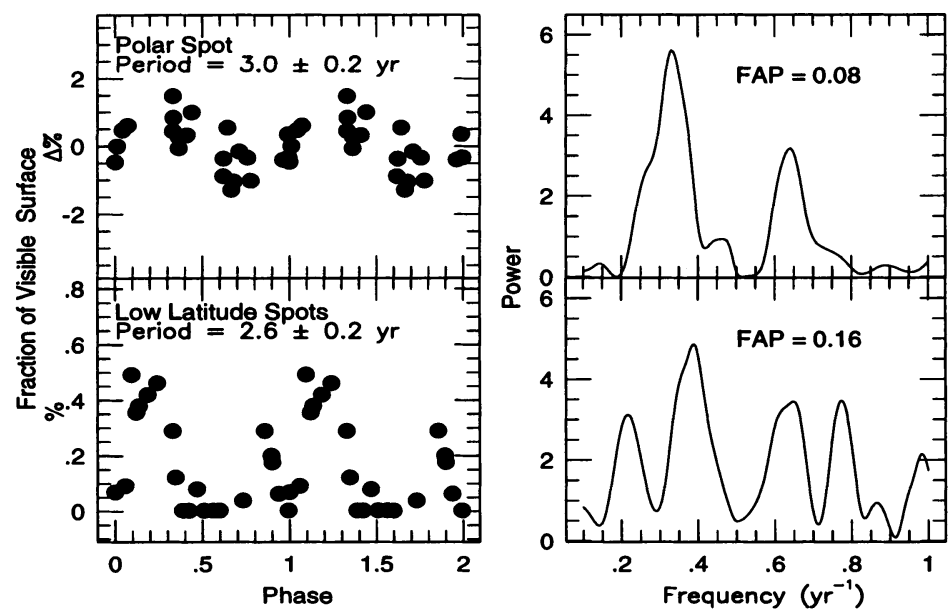

Figure 7. (left) The percent change in the area of spots phased to a period of 3.0 years. (right) Periodogram results of polar (upper) and low-latitude (lower) spot areas.

a FAP of 0.16 . The lower left corner of Figure 7 shows the low-latitude spot phased to the 2.6-yr period. The quality of the data is probably not sufficient to determine if the variations of the polar spot and low-latitude spots indeed have different periods. (The best phase diagrams of the polar and low-latitude spot areas are provided by the respective periods of the periodograms.) At this point, we can only conclude that both the polar feature and low-latitude spots may show marginal evidence for an $\approx 3$-year periodicity in the variations of their total areas. 


\section{Discussion}

Clearly the most prominent feature of our images of HR 1099, and a recurring theme in other's images of rapidly-rotating spotted stars is the ubiquitous large cool polar spot. Since the reality of large cool polar spots is still not universally accepted by the cool-star community, a few words about this seem in order here. Perhaps the best argument for their reality is that we do not see them on some more moderately-rotating RS CVn stars, for example $\sigma$ Gem (Hatzes 1993) nor do we ever see them on other types of stars we have imaged (Ap stars). In some other cool-star cases, like ZAMS Pleiades stars (Stout and Vogt 1995), they are at high latitudes, but are not symmetrically placed on the pole as would be expected if they were some artifact of our inability to model line profiles properly. We also recently completed a study (Hatzes et al. 1995) in which we showed that the inclination dependence of line core flattening in a sample of stars was not explainable by gravity darkening, by differential rotation, or by a bright equatorial band, but was quite consistent with the inclination aspect-dependence produced by a polar dark spot. Other researchers have also found these polar spots both in intensity images and in Stokes parameter imagery. Finally, phaseindependent $\mathrm{TiO}$ features in the spectra of some low-inclination spotted stars also strongly support the presence of large cool polar spots.

Such permanent cool polar spots must, if we've learned anything from the magnetic fields of sunspots, be telling us that there are multi-kgaussstrength fields filling these polar spots and suppressing convection in the photosphere to a degree that the surface is cooled sufficiently to produce visible spots, just as occurs in sunspot umbrae. However, from the standpoint of locations, areas, shapes, and differential rotation, starspots look much more like solar coronal holes than giant sunspots. In fact, the resemblance between the polar spot of HR 1099 and its variable 'protuberances' with the solar polar coronal hole and its 'polar hole extensions' is quite striking. Both straddle the pole and have similar areas and shapes.

Starspots also show surprisingly little differential rotation, quite unlike sunspots, but again very similar to solar coronal holes. On the Sun, coronal holes are the footprints of the unipolar magnetic regions (UMR's) of the global coronal field, and their shapes are maintained rigidly (in the face of considerable photospheric shear) by a few-gauss current-free global dipole coronal field (Wang and Sheeley 1993), the same dipole field which produces the polar coronal holes. The differential rotation of solar coronal holes is governed by the stability of the globally-averaged current-free coronal field rather than by the latitudinal shear of the solar photosphere. If the solar coronal hole analogy holds true for starspots, then the differential rotation derived from starspots on RS CVn stars may actually be revealing very little 
about the latitudinal shear of the photosphere, but rather may be more a consequence of the stability of a powerful, current-free global dipole field. This global field is probably of multi-kgauss-strength at the pole (and only half as strong at the equator if dipolar), orders of magnitude stronger than the few-gauss global fields of the Sun. Presumably, both the strength and stability of this global field increases with stellar angular rotation velocity (i.e. dynamo strength) thereby offering a simple explanation for why the observed differential rotation decreases as angular velocity increases annong active stars.

That large polar spots are a quite common feature of rapidly rotating spotted stars, perhaps suggests that there is a large polar spot at both poles of the star. If so, it becomes reasonable to assume that these UMR polar spots are of opposite polarity and their field lines connect out beyond the Alfven radius. These polar spots thus probably bear witness to a strong dipole or low-order magnetic field within the star which is aligned with the star's rotation axis. Whether this axisymmetric field is produced solely by an axisymmetric dynamo and/or is the result of accumulation of flux from non-axisymmetric dynamos is not clear. Perhaps both contribute. Indeed, the track of Feature B may be showing us this winding up of field lines around the pole into the toroidal magnetic structure seen by Donati et al. (1992).

We conclude from our Doppler imaging studies that the starspots on HR 1099 and many other rapidly-rotating late-type stars are essentially powerful stellar analogs of solar coronal hole structures (UMR's), but formed by multi-kgauss strength magnetic fields rather than the few-gauss global coronal fields of the Sun. The persistent polar spots and increasing stability of spot features with increasing rotation velocity suggests to us that rapidly rotating and highly convective stars with prominent polar spots have probably wound up multi-kgauss axisymmetric dipole fields. Such strong dipolar fields could certainly have escaped previous direct detection. Any purely axisymmetric field component would show no rotational modulation. Also, most of the magnetic flux would be quite effectively hidden from detection in the optical region of the spectrum by being concentrated in the very cool (and hence optically dark) polar spots, but is easily detectable indirectly via Doppler images of these ubiquitous polar spots. These very active stars are also quite rapid rotators, and their rotational line broadening makes Zeeman-splitting detections difficult. However, recent magnetic measurements in the infrared (Saar 1995), where the contrast between spot and photosphere is much less, may finally now be directly detecting these strong fields in the polar regions of some active stars.

HR 1099 tells us that at least some of the magnetic flux which emerges at lower latitudes migrates pole-ward to join with the polar spot. A polar 
starspot is thus probably the equivalent of the solar polar coronal hole, complete with variable 'protuberances' (polar hole extensions) but filled with multi-kgauss-strength magnetic fields similar to that found in sunspot umbrae. The stability (differential rotation) of spots is probably maintained by this powerful current-free global dipole field and, in HR 1099, this dipole field is apparently tightly synchronized to the orbit. Given that this dipole probably penetrates through to the core of the star, this is not altogether surprising. The dipole is probably rooted to the rotatior: rate of a core which was long ago synchronized to the orbit, and remains so today, even after the outer layers of the star expanded (and perhaps slowed) as the star left the main sequence.

We thus propose the following simple two-pronged analogy of starspots to solar activity. Starspots are much like sunspot umbrae by virtue of their large (multi-kgauss) field strengths, which are strong enough to inhibit convection and cause a large enough temperature drop in the photosphere to be easily visible in the optical. However, as regards their global properties (sizes, shapes, locations, differential rotation, and migration), they are a direct analog of solar coronal holes (UMR's), with their structure and evolution heavily influenced by a strong axisymmetric multi-kgauss dipole field which has been built up within the star.

While HR 1099 reveals evidence for spots emerging at low latitude and migrating pole-ward (at a latitude migration rate of $6-30 \mathrm{~m} / \mathrm{s}$ ) to eventually merge with the polar spot, we don't yet know whether these spots reinforce the polar spot with like-polarity flux, or instead destroy it in some sort of feedback process which may ultimately produce a periodic polarity-reversal of the polar spot. Whichever the case, magnetic flux which emerges at lower latitudes probably separates out into unipolar magnetic regions, and then migrates pole-ward to merge, either constructively or destructively, with the polar spot. The axisymmetric dipole field within HR 1099, if related to polar spot area, may show marginal evidence for $\approx 3$-year periodicity (i.e. a dynamo cycle), but probably varies only weakly, if at all, in total strength.

As shown theoretically (Moss et. al. 1995) from mean field dynamo models, these stars probably excite both axisymmetric and non-axisymmetric dynamo fields. The models show that, at moderate values of the Taylor number, stable non-axisymmetric fields (perpendicular-dipole topology) can be excited in deep convective shells, and this flux rises to the surface, emerging at intermediate-latitudes to form spots. These theoretical studies apparently have some difficulty producing flux emergence at low latitudes, but should be pursued to see if they can produce flux emergence at even lower latitudes since HR 1099 frequently forms spots even at the equator. At large Taylor numbers (large differential rotation), only axisymmetric dynamo solutions are stable, and one would therefore expect the axisymmetric 
field (i.e. polar spots) to become more prominent with increasing angular velocity. There may now actually be observational support for this. The more slowly-rotating RS CVn stars (Hatzes 1993) exhibit only intermediatelatitude spots, presumably created from such non-axisymmetric dynamos, and do not exhibit the permanent polar spot signature of an axisymmetric dynamo mode.

The large polar starspots and low-latitude spots on HR 1099 and other rapidly rotating stars probably indicate that both types of dynamos are excited within these stars, and furthermore that the flux contribution from the axisymmetric component increases with angular rotation velocity, resulting in more prominent and long-lived polar spots. Some of the flux which emerges at low and intermediate latitudes from the less stable nonaxisymmetric dynamo modes clearly makes its way to the poles on HR 1099 and there may either simply accumulate to reinforce the axisymmetric dipole field, or may erode it if of opposite polarity, perhaps as part of a long-term polarity-reversing periodic process, as occurs on the Sun. Further Zeeman Doppler imagery, particularly in the infrared, of stars with polar spots could be very helpful in sorting out this question.

Based solely on the Solar coronal field analogy, and on the efficiency of the axisymmetric dynamo mode, we suspect that, close to the pole, the field assumes the topology of an axisymmetric dipole. But perhaps the field topology at lower latitudes within the polar spot is simply a continuation of the toroidal pattern detected around the polar spot periphery by Donati et al. (1992). If so, as perhaps also suggested by the track of Feature B on HR 1099 , magnetic flux is literally 'winding up' around the pole like spaghetti around a fork. The field strength would thus increase with latitude, and one would be able to detect such toroidal fields only up to a latitude where their strength was sufficient to suppress convection (i.e.. the edge of the spot). This would produce a strong bias for field detection precisely at the boundary of the polar spot, just as observed. That latitude is exactly where the fields would be the strongest before becoming invisible within the spot. At lower latitudes, the fields would be too weak to detect. Above that latitude, the photosphere would then turn dark, forming the polar spot, and the tightly-coiled fields within the polar spot would be effectively hidden from view.

Clearly the axisymmetric and non-axisymmetric dynamo modes need to be further explored through realistic dynamo modeling. The relative contributions of each should be predicted as a function of rotation period, and can then be directly checked through Doppler images of stars over a range of rotation periods. HR 1099 suggests that both modes are probably active in the rapidly rotating RS CVn stars, and furthermore that there may be a pronounced winding of low-latitude emergent flux into toroidally-wrapped 
flux up near or even in the polar spot. Does this winding of flux continue right up to the polar singularity, or is this toroidal flux being wound tightly around an axisymmetric dipole field? In either case, such complex nonpotential field configurations might well be expected to give rise to stable high temperature/energetic phenomenon within or above the polar spot. The details of the interaction between axisymmetric and non-axisymmetric dynamo modes must be quantified, with the aim of understanding how these modes interact over the long term, to see if they can indeed build up sizeable polar fields, and if this field is stable in strength/polarity, or if it varies periodically. It is an area of theoretical exploration now ripe for exploration, and its predictions can be sensibly tested on spotted stars through Doppler imagery.

Finally, the large cool polar spots on HR 1099 are presumably a direct look at the 'magnetic brake' which is ultimately responsible for slowing the rotation of many rapidly-rotating single late-type stars. The tidallylocked spotted subgiant star in the HR 1099 binary system is thus probably 'standing on the brakes', but alas in vain in the face of tidal locking to the large angular momentum reservoir of the orbit.

\section{References}

Andrews A.D., Rodono M., Linsky J.L., Brown A., Butler C.J., Catalano S., Scaltriti F., Busso M., Nha I., Oh J.Y., Henry M.C.D., Hopkins J.L., Landis H.J., Engelbrektson S., 1988, A\&A 204, 177.

Donati J.-F., Semel M., Rees D.E., Taylor K., Robinson R.D., 1990, A\&A 232, L1.

Donati J.-F., Brown S.F., Semel M., Rees D.E., Dempsey R.C., Matthews J.M., Henry G.W., Hall D.S., 1992, A\&A 265, 682.

Hall D.S., 1991, in The Sun and Cool Stars: Activity, Magnetism, and Dynamos, I. Tuominen, D. Moss, and G. Rüdiger (eds.), Berlin:Springer-Verlag, p. 353.

Hatzes A.P., 1993, ApJ 410, 777.

Hatzes A.P., Vogt S.S., Ramseyer T.F., Misch T., 1995, in Strassmeier K.G. (ed.), Poster Proceedings: Stellar Surface Structure, Univ. Vienna, Vienna, p. 9

Kang Y.W., Wilson R.E., 1989, AJ 97, 848.

Mohin S., Raveendran A.V., 1993, A\&AS 100, 331.

Moss D., Barker D.M., Brandenburg A., Tuominen I., 1995, A\&A 294, 155.

Rodono M., Cutispoto G., Pazzani S., Catalano S., Byrne P.B., Doyle J.G., Butler C.J., Andrews A.D., Blanco C., Marilli E., Linsky J.L., Scaltriti F., Busso M., Cellino A., Hopkins J.L., Okazaki A., Hayashi S.S., Zeilik M., Helston R., Henson G., Smith P., Simon T., 1986, A\&A 165, 135.

Saar. S.H. 1995, these proceedings.

Schüssler M., Solanki S.K., 1992, A\&A 264, L13.

Stout N., Vogt S.S., 1995, these proceedings.

Vogt S.S., Penrod G.D., Hatzes A.P., 1987, ApJ 321, 496.

Vogt S.S., Hatzes A.P., Misch A.T., Kürster M., 1996, ApJS, in preparation.

Wang Y.-M., Sheeley N.R. Jr., 1993, ApJ 414, 916.

Zhai D.S., Foing B.H., Cutispoto G., Zhang R.X., Catala C., Char S., Zhang X.B., Jankov S., 1994, A\&A 282, 168. 